\title{
OPEN Metabolomics for early detection of stress in freshwater alga Poterioochromonas malhamensis exposed to silver nanoparticles
}

\author{
Wei Liu $\circledast^{1}$, Sanghamitra Majumdar $\circledast^{2}$, Weiwei Li ${ }^{2}$, Arturo A. Keller $\circledast^{2}$ \& \\ Vera I. Slaveykova ${ }^{1 凶}$
}

Silver nanoparticles (AgNPs) are one of the most used engineered nanomaterials. Despite progress in assessing their environmental implications, knowledge gaps exist concerning the metabolic perturbations induced by AgNPs on phytoplankton, essential organisms in global biogeochemical cycles and food-web dynamics. We combine targeted metabolomics, biouptake and physiological response studies to elucidate metabolic perturbations in alga Poterioochromonas malhamensis induced by AgNPs and dissolved Ag. We show time-dependent perturbation of the metabolism of amino acids, nucleotides, fatty acids, tricarboxylic acids, photosynthesis and photorespiration by both Ag-treatments. The results suggest that dissolved $\mathrm{Ag}$ ions released by AgNPs are the major toxicity driver; however, AgNPs internalized in food vacuoles contributed to the perturbation of amino acid metabolism, TCA cycle and oxidative stress. The metabolic perturbations corroborate the observed physiological responses. We highlight the potential of metabolomics as a tool for understanding the molecular basis for these metabolic and physiological changes, and for early detection of stress.

Metabolomics tracks the changes in low-molecular-weight metabolites involved in different biological reactions $s^{1,2}$ under different environmental stimuli and stressors. It is well suited to study organism-environment interactions ${ }^{3}$ and serves to integrate information on metabolic pathway perturbation with physiological responses to stressors. Nanoparticle-induced metabolic perturbations to environmentally relevant organisms, such as plants, have only been recently studied systematically by both untargeted and targeted metabolomics ${ }^{4-9}$. The perspectives and challenges in the application of metabolomics to understand the nanotoxicology of plants have recently been comprehensively reviewed ${ }^{10,11}$. Regarding phytoplankton, only one metabolomic study focused on the effect of silver nanoparticles (AgNPs) on cyanobacterium Microcystis aeruginosa ${ }^{12}$. Hence, there is a significant knowledge gap with respect to the metabolic perturbations induced by engineered nanomaterials on phytoplankton, despite their pivotal role in global biogeochemical cycles and food-web dynamics ${ }^{13}$.

The present study focusses on AgNPs as they are one of the most extensively used materials in a variety of consumer products, mainly due to their biocidal properties ${ }^{14}$. The release of AgNPs from different products ${ }^{15}$ has raised significant concerns about possible consequences to the aquatic environment ${ }^{16}$. Indeed, the potential of AgNPs to affect phytoplankton community structure and functioning ${ }^{17,18}$, as well as individual phytoplankton species $^{19,20}$ is well documented. Dissolution, uptake, oxidative stress ${ }^{21,22}$ and photosynthesis inhibition ${ }^{17,19,23}$ were found as the major drivers of AgNP toxicity. However, the extent of uptake and dissolution of AgNPs, and their contribution to the overall toxicity in phytoplankton community remains unclear. For example, released Ag ions rather than AgNPs were responsible for the toxicity to green algae Raphidocelis subcapitata ${ }^{24}$ and Chlamydomonas reinhardtii ${ }^{25}$; however, AgNPs directly contributed to the toxicity in chrysophyte Ochromonas dania ${ }^{26}$. AgNPs were also found in the cytoplasm of $C$. reinhardti ${ }^{27}$, but the possible alterations of algal metabolism were not explored. While it is useful to study the physiological responses of AgNPs, there is a need to understand how AgNPs affect the metabolic processes of these organisms. Hence, metabolomics can serve to reveal these subtle but very important effects.

\footnotetext{
${ }^{1}$ Department F.-A. Forel for Environmental and Aquatic Sciences, Environmental Biogeochemistry and Ecotoxicology, Faculty of Sciences, Earth and Environment Sciences, University of Geneva, Uni Carl Vogt, 66 Blvd Carl-Vogt, 1211 Geneva, Switzerland. ${ }^{2}$ Bren School of Environmental Science and Management, University of California, Santa Barbara, CA 93106-5131, USA. ${ }^{\square}$ email: vera.slaveykova@unige.ch
} 
A

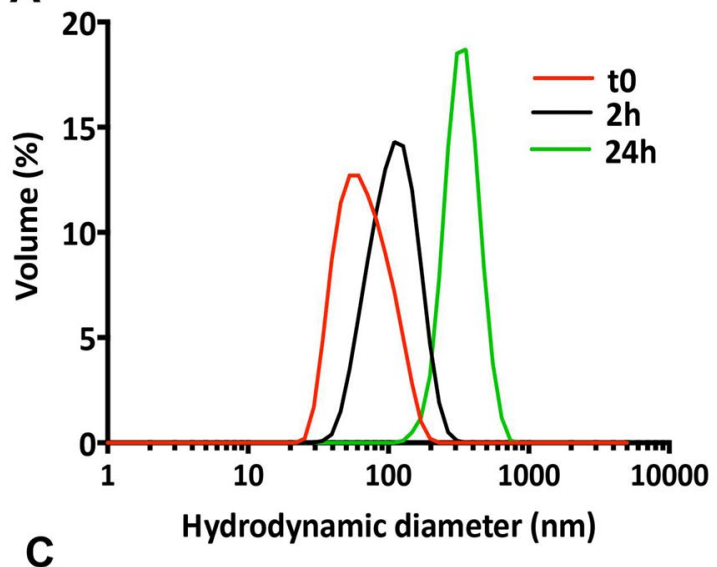

B

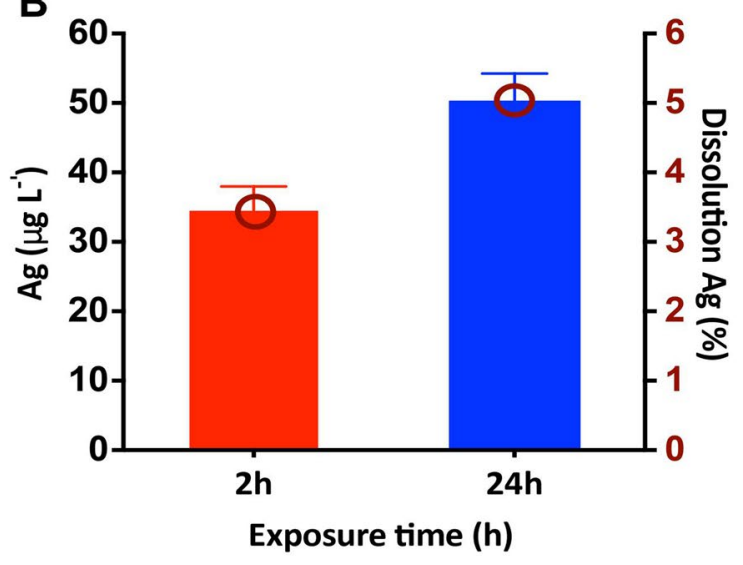

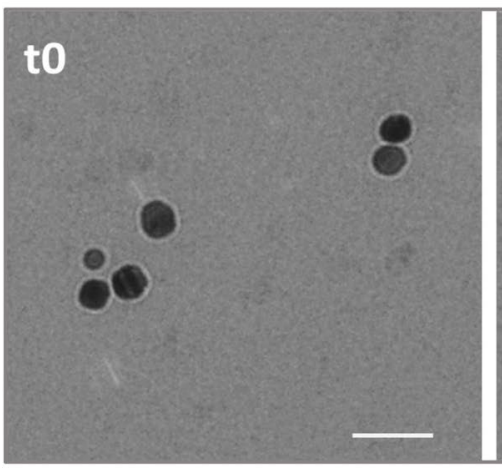
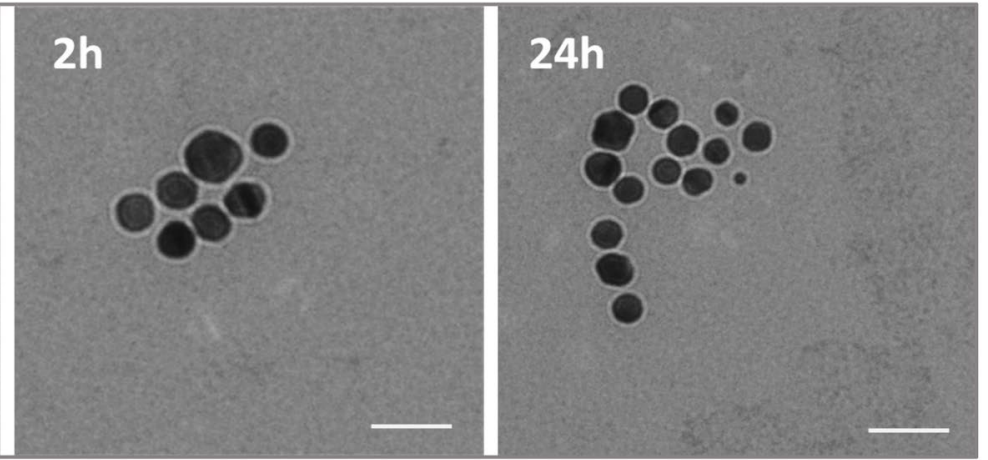

Figure 1. Characterization of the suspensions of AgNPs in the exposure medium at t0, $2 \mathrm{~h}$ and $24 \mathrm{~h}$. (A) AgNPs hydrodynamic size distribution obtained by DLS as volume \%; (B) AgNPs dissolution in Waris-H medium. Concentration and percentage of dissolved Ag in $1 \mathrm{mg} \mathrm{L}^{-1}$ AgNP suspensions; (C) TEM images of AgNPs in the exposure medium at t0, $2 \mathrm{~h}$ and $24 \mathrm{~h}$. Scale bars: $50 \mathrm{~nm}$.

The primary goal of the current study is to obtain new insights into metabolic perturbations underlying the cellular responses in algae exposed to AgNPs. Using targeted metabolomics we address the following research questions: (1) what are the major metabolic pathways of algae that are influenced by AgNPs?; (2) what is the time progression of the metabolic perturbations?; (3) are they common or different for AgNPs and dissolved Ag treatments?

We examine the interactions of citrate-coated $20 \mathrm{~nm}$ AgNPs with the freshwater alga Poterioochromonas malhamensis, as representative of freshwater phytoplankton. This golden-brown alga often dominates mixotrophic phytoplankton populations ${ }^{28}$. P. malhamensis uses a wide selection of different organic food sources via phagotrophy or osmotrophy ${ }^{29}$. Hence, we assume this alga can take up AgNPs and their aggregates via phagocytosis. It also plays an important role in the fate and transport of organic matter in the environment ${ }^{30,31}$. Citrate-coated AgNPs were chosen in the present study according to the recommendations of the Organization for Economic Cooperation and Development ${ }^{32}$. Liquid chromatography-mass spectrometry (LC-MS)-based targeted metabolomics was used to quantify selected primary and secondary metabolites and their modulation by AgNPs and dissolved Ag treatments. In parallel, physiological responses such as enhanced generation of reactive oxygen species (ROS), lipid peroxidation and changes in photosynthetic activity were assessed. Uptake of AgNPs by $P$. malhamensis, together with key characteristics of AgNPs in the exposure medium were determined. The results highlight the high added value of metabolomics to elucidate the mechanisms that cause the physiological response in phytoplankton induced by stressors such as engineered nanomaterials.

\section{Results and discussion}

Characterization of AgNPs suspensions in the exposure medium. The suspensions of AgNPs were characterized in terms of hydrodynamic size distribution and average diameter, zeta potential (ZP) and dissolution. Dispersion of $1 \mathrm{mg} \mathrm{L}^{-1}$ AgNPs in the exposure medium, resulted in an immediate formation of aggregates with a peak of the hydrodynamic size distribution centered at $61 \pm 5 \mathrm{~nm}$, and $\mathrm{ZP}$ of $-23 \pm 3 \mathrm{mV}$. The size distributions shifted towards higher values with a maximum at $127 \pm 6 \mathrm{~nm}(\mathrm{ZP}:-20 \pm 2 \mathrm{mV})$ at $2 \mathrm{~h}$ and $350 \pm 3 \mathrm{~nm}$ (ZP: $-12 \pm 2 \mathrm{mV}$ ) at $24 \mathrm{~h}$ (Fig. 1A). These results indicate that the AgNPs aggregated in the exposure medium with time. The above results are consistent with transmission electron microscopy (TEM) observations showing spherical AgNPs with a size of $20 \mathrm{~nm}$ and aggregated forms (Fig. 1C). 


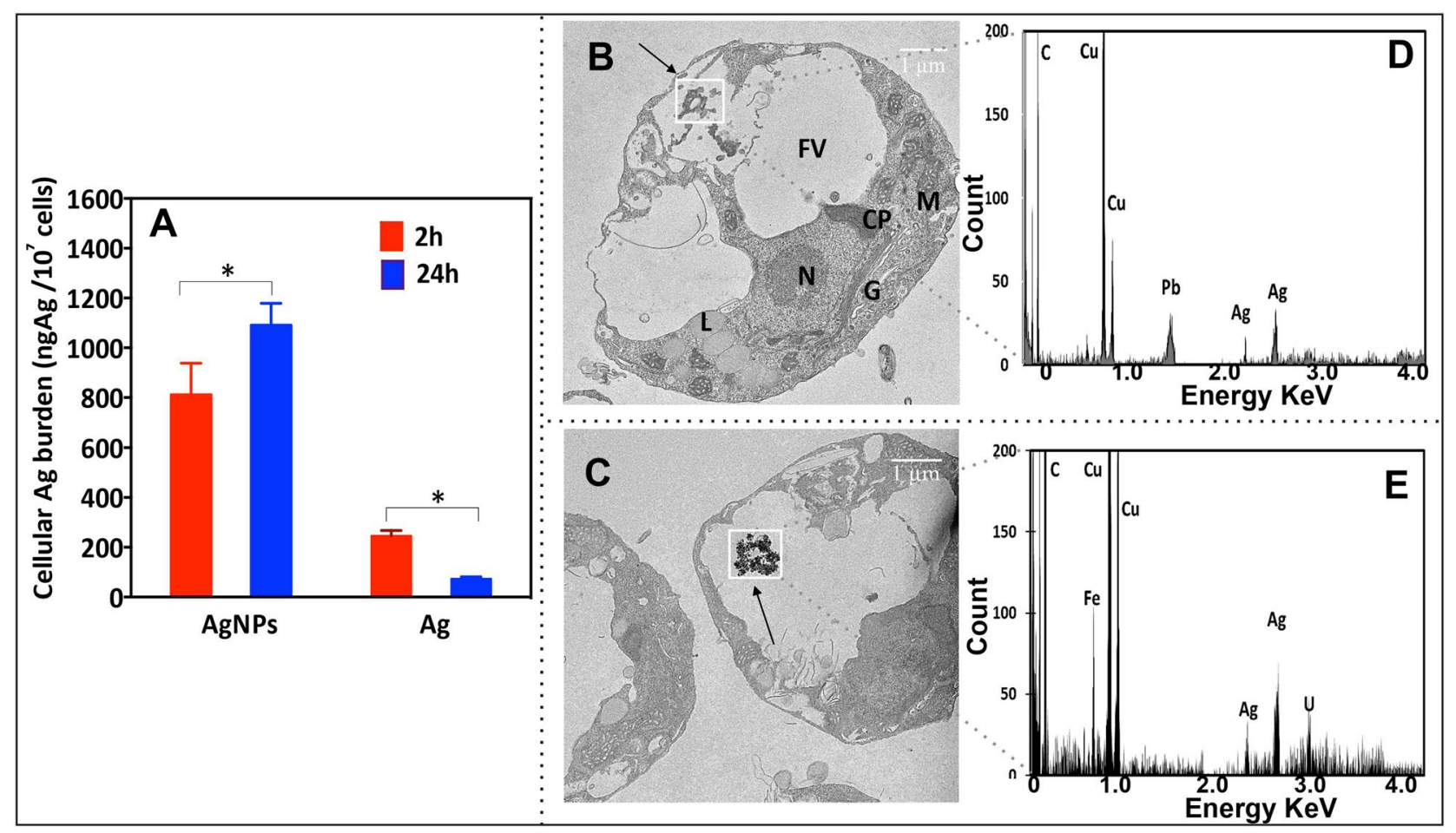

Figure 2. (A) Ag cellular burden of P. malhamensis exposed to $1.0 \mathrm{mg} \mathrm{L}^{-1} \mathrm{AgNPs}$ or $40.7 \mu \mathrm{g} \mathrm{L}^{-1} \mathrm{AgNO}_{3}$ during $2 \mathrm{~h}$ and $24 \mathrm{~h}$. The results are presented as ng of cellular Ag per $1 \times 10^{7}$ cells. Asterisk indicates a significant difference between treatments obtained by two-way analysis of variance (ANOVA) followed by a Sidak's multiple comparisons test $(p<0.05)$; (B,C) TEM images of $P$. malhamensis: treated with $1 \mathrm{mg} \mathrm{L}^{-1} \mathrm{AgNPs}_{\mathrm{g}}$ for $2 \mathrm{~h}$ (B) and for $24 \mathrm{~h}(\mathbf{C})$; (D,E) energy dispersive X-ray (EDX) spectra correspond to the interest area from C (D) and D (E). Letters on (B) denote cell organelles: $G$ Golgi body, $M$ mitochondrion, $N$ nucleus, $F V$ food vacuole, $L$ lipid drops, $C P$ chloroplast. ${ }^{\star} p<0.05,{ }^{* *} p<0.01,{ }^{* *} p<0.001$.

Dissolution of the AgNPs was low with dissolved Ag representing 2.50\% and 4.07\% of the total Ag in the suspensions after $2 \mathrm{~h}$ and $24 \mathrm{~h}$, respectively (Fig. 1B). These values were comparable with previously reported results in other exposure media specific to algal bioassays $\mathrm{s}^{25,26,33-35}$.

Ag cellular burden and internalization of AgNPs by P. malhamensis. To quantify cellular Ag cellular burden and explore the internalization of AgNPs, we combined ICP-MS analysis of the algal cells for total cellular Ag concentrations and 2D TEM of the ultrathin cell sections. Exposure to AgNPs led to a significant accumulation of Ag in the algal cells, which increased with exposure time (Fig. 2A). Silver cellular burden was higher in AgNP treatments compared with exposures to $\mathrm{AgNO}_{3}$, and increased with exposure duration from 2 to $24 \mathrm{~h}$. The contribution of the dissolved Ag present in the suspensions of AgNPs to the overall Ag was low (Fig. 2A). Ag cellular burden of P. malhamensis exposed to dissolved Ag at $24 \mathrm{~h}$ was lower than at $2 \mathrm{~h}$, which could be related to decrease in the exposure medium, following significant cellular accumulation, as well as "growth-dilution effect" a kinetic effect in which accumulated metals are diluted within the algal cell by photosynthetically fixed carbon ${ }^{36}$.

TEM revealed the presence of aggregates with size around $100 \mathrm{~nm}$ in the food vacuoles of $P$. malhamensis exposed to AgNPs for $2 \mathrm{~h}$ (Fig. 2B). The above observation was confirmed in the $24 \mathrm{~h}$ exposure, where even larger aggregates of around $500 \mathrm{~nm}$ were found in the vacuoles (Fig. 2C). The characteristic peak of $\mathrm{Ag} \mathrm{L}_{\alpha 1}$ and $\mathrm{L}_{\beta 1}$ observed in EDX spectra confirmed that the aggregates contained Ag (Fig. 2D,E). The present results revealed that $P$. malhamensis internalized AgNPs, probably by phagocytosis. Indeed $P$. malhamensis is known to ingest plankton by phagocytosis ${ }^{37}$. Hence, the algae could possibly misidentify AgNPs and their aggregates as nutritive particulate organic matter and thus ingest them in a similar way. The above results are consistent with the observation that AgNPs are internalized in the cells of another species, Ochromonas danica ${ }^{26}$, which has been previously shown to have endocytosis ${ }^{31}$. However, it remains unclear whether AgNPs inside the cells alter algal metabolism directly or indirectly by the release of Ag ions.

Effect of Ag-treatments on algal physiology. Exposure to AgNPs resulted in a significant and timedependent increase of cellular ROS generation. The percentage of cells with enhanced ROS production increased to $2.1 \pm 0.3$ at $2 \mathrm{~h}$ and $4.7 \pm 0.5$ times at $24 \mathrm{~h}$ exposure, in comparison with unexposed controls (Fig. $3 \mathrm{~A}$ ). AgNP concentration-dependent increase of the percentage of cells with excessive ROS was also evidenced (see supplementary information). These results are consistent with the enhanced ROS generation observed in Chlorella vulgaris and Dunaliella tertiolecta exposed to $\mathrm{AgNPs}^{38}$. Exposure to $\mathrm{AgNO}_{3}$ at a concentration corresponding to 

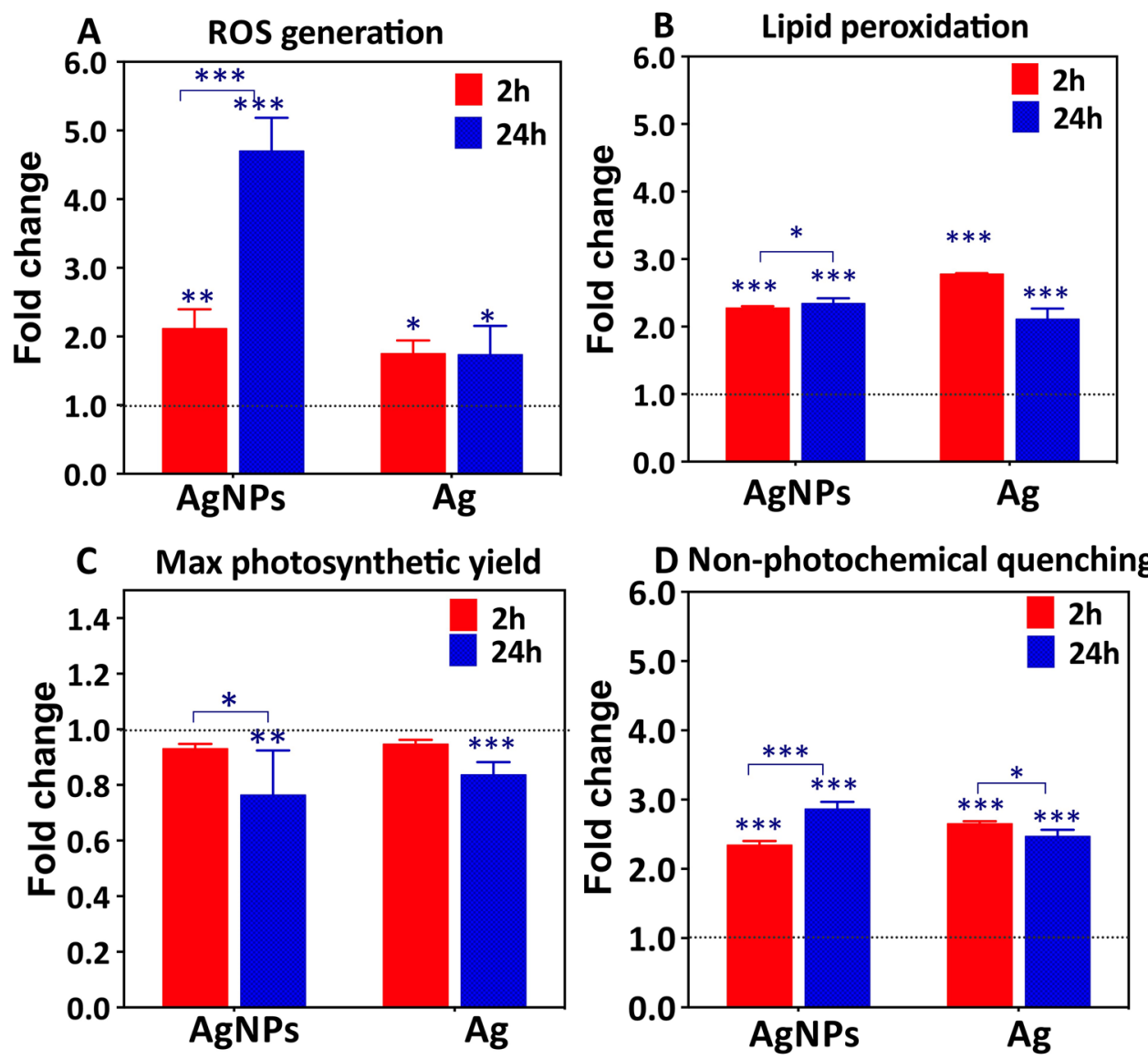

Figure 3. Effect of $1.0 \mathrm{mg} \mathrm{L}^{-1} \mathrm{AgNPs}$ or $40.7 \mu \mathrm{g} \mathrm{L}^{-1} \mathrm{AgNO}_{3}$ exposure on physiology of P. malhamensis. (A) ROS generation, determined by CellRoxGreen stain and FCM; (B) lipid peroxidation, assessed by MDA test; (C) maximum quantum yield of photosystem II (Fv/Fm); and (D) non-photochemical quenching (NPQ). Fold change is calculated as a ratio of the respective effects observed in $\mathrm{Ag}$ - treated cells and untreated control. Asterisks indicate a significant difference between treatments obtained by two-way analysis of variance (ANOVA) followed by a Sidak's multiple comparisons test: ${ }^{*} p<0.05 ;{ }^{* *} p<0.01 ;{ }^{* *} p<0.001$.

the dissolved Ag in the AgNP suspensions resulted in about 1.8 \pm 0.2 time increase of cells with enhanced ROS, which was time independent. The results also revealed that AgNPs, rather than dissolved Ag present in the AgNP suspensions, contributed significantly to cellular ROS generation, particularly at $24 \mathrm{~h}$ exposure (Fig. 3A). Excessive levels of ROS could lead to cellular oxidative stress and damage such as lipid peroxidation ${ }^{22}$. Indeed, both Ag-treatments induced lipid peroxidation in P. malhamensis (Fig. 3B).

Exposure to AgNPs resulted in about $2.27 \pm 0.03$ times increase of lipid peroxidation at $2 \mathrm{~h}$ as compared with unexposed controls, which was unchanged at $24 \mathrm{~h}$ exposure $(2.34 \pm 0.07$ times increase). Exposure to dissolved $\mathrm{Ag}$ resulted in $2.78 \pm 0.10$ times increase in the cells with lipid peroxidation at $2 \mathrm{~h}$ and $2.11 \pm 0.15$ times at $24 \mathrm{~h}$, as compared with unexposed controls. Comparable influence of AgNPs and dissolved Ag on the lipid peroxidation suggests that the dissolved Ag present in the AgNPs suspensions drives the observed effect. Lipid peroxidation is considered a marker of oxidative stress damage ${ }^{39}$. Severe lipid peroxidation could lead to structural change in membranes, impaired membrane fluidity and canals, altered signaling proteins linked to membranes, and increased membrane permeability to ions ${ }^{40}$.

The maximal quantum yield (Fv/Fm), used as a measure for the efficiency of PSII was significantly decreased after $24 \mathrm{~h}$ exposure to AgNPs or dissolved Ag, while at $2 \mathrm{~h}$ the Fv/Fm were comparable with those of the untreated controls (Fig. 3C). The fold change of Fv/Fm decreased from $0.93 \pm 0.02$ at $2 \mathrm{~h}$ to $0.76 \pm 0.16$ at $24 \mathrm{~h}$ exposure to AgNPs. The electron transport rate of PS II is also affected since it is proportional to the quantum yield ${ }^{41}$. The above finding is consistent with the existing literature showing a significant decrease of the photosynthetic yield of alga Isochrysis galbana exposed to high concentrations of $\mathrm{AgNPs}^{42}$ as well as phototrophic plankton communities $^{43}$. The inhibition of PSII can be explained by the sensitivity of the photosynthetic machinery to $\mathrm{Ag}^{+}$. Indeed, $\mathrm{Ag}^{+}$was shown to bind to thiols of functional proteins, displacing $\mathrm{Cu}^{+}$in the proteins and causing disturbance or inactivation of the photosynthetic electron transport of the photosystem in a green alga Chlamydomonas reinhardtii ${ }^{4}$. In addition, about two-fold increase of non-photochemical quenching (NPQ) by both Ag-treatments was observed (Fig. 3D). The fold change of NPQ were $2.34 \pm 0.06$ and $2.86 \pm 0.10$ at 2 and $24 \mathrm{~h}$-exposure to AgNPs with respect to untreated controls. NPQ is the degree to which photons are lost in the photosynthetic process, therefore the energy absorbed is divided between the fraction used in photochemistry 

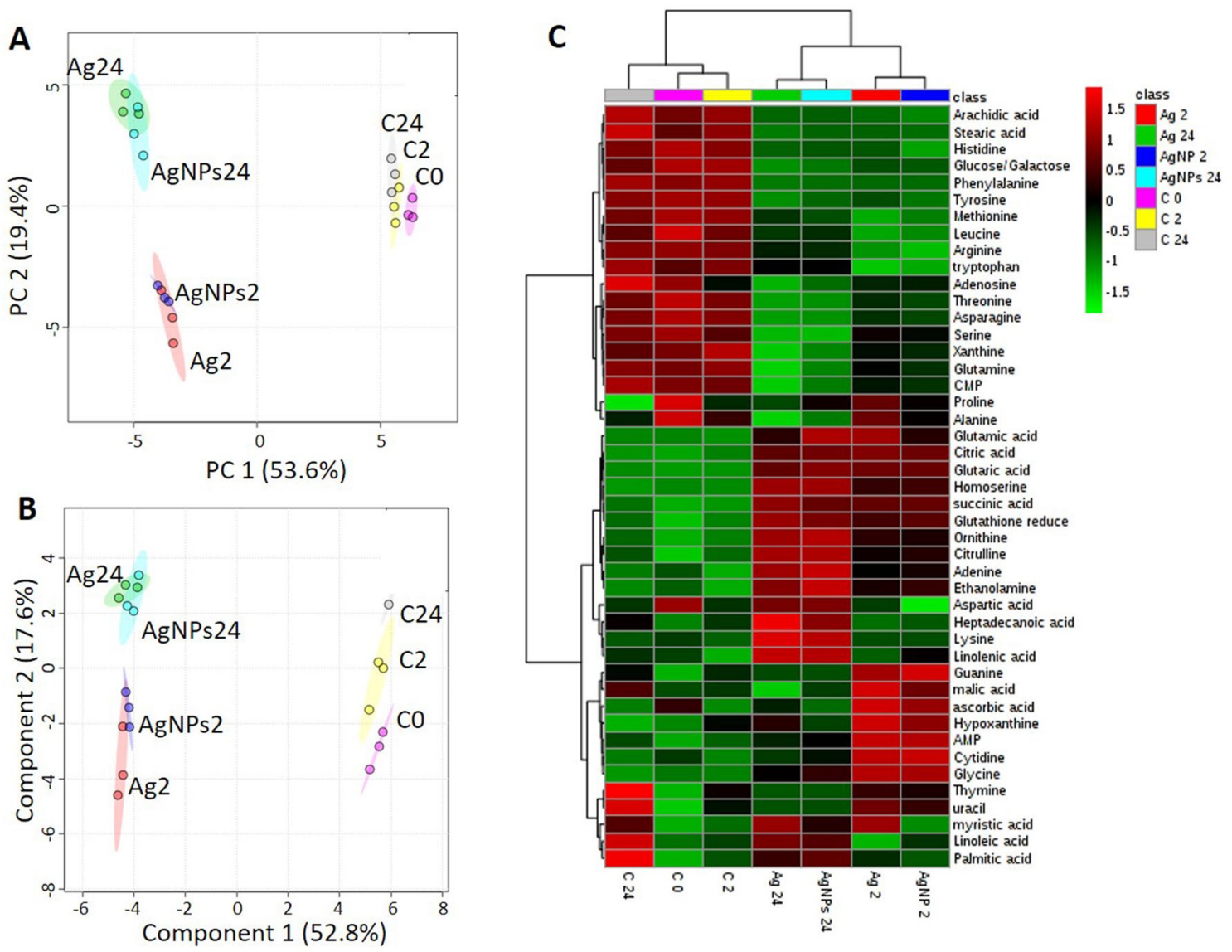

Figure 4. (A) Principal component analysis (PCA) and (B) partial least-squares discriminate analysis (PLS-DA) score plots of metabolic profiles in P. malhamensis treated with AgNPs and dissolved Ag, and untreated controls. (C) Clustering metabolites and samples shown in a heat map (Euclidean distance and Ward clustering algorithm). Data were normalized by using probabilistic quotient normalization by untreated control group at time $0(\mathrm{C} 0), \log$ transformed and autoscaled. Exposure to $40.7 \mu \mathrm{g} \mathrm{L}^{-1} \mathrm{AgNO}_{3}$ for $2 \mathrm{~h}(\mathrm{Ag} 2)$ and $24 \mathrm{~h}$ (Ag24); exposure to $1 \mathrm{mgL}^{-1} \mathrm{AgNPs}$ for $2 \mathrm{~h}$ (AgNPs2) and for 24 (AgNPs24); C2: unexposed controls at time $2 \mathrm{~h}$; C24: unexposed controls at time $24 \mathrm{~h}$. The score plots and heatmap are generated by MetaboAnalyst 4.0 (https:// www.metaboanalyst.ca/ $)^{72}$.

and that lost non-photochemically. Phytoplankton can regulate the light-harvesting domain, increase the amount of energy dissipated as heat via NPQ, thus protecting the cells from oxidative stress ${ }^{45}$. These results showed that the photosynthetic performance of $P$. malhamensis at $24 \mathrm{~h}$ was strongly affected by AgNPs and dissolved Ag.

Metabolic response of $P$. malhamensis to AgNPs and dissolved Ag. A total of 94 metabolites were considered, representing major groups of primary metabolites, including antioxidants, amines, amino acids, organic acids/phenolics, nucleobases/sides/tides, sugars/sugar alcohols and fatty acids. Among them 52 were quantified in different Ag-treatments and controls. A general overview of the treatment clustering was obtained by the unsupervised PCA, supervised PLS-DA and clustering analysis (Fig. 4).

The PCA score plot (Fig. 4A) demonstrated very good separation of both Ag-treatments and unexposed controls, along the first principal component (PC1), which explained 53.6\% of the total variance. Excellent separation was found between 2 and 24 h exposures, underlying the importance of tracking the evolution of the metabolic response with time. The PLS-DA score plot (Fig. 4B) confirmed a distinction between Ag-treatments at $2 \mathrm{~h}$ and $24 \mathrm{~h}$ and untreated samples. However, no noticeable separation was observed between AgNPs and dissolved Ag-treatments for a given exposure time. The responsive metabolites were subsequently discriminated on the basis of VIP score $>1$ (Fig. S1).

Twenty-seven important features identified by PLS-DA allowed a good separation between both AgNPs and Ag-treated samples and untreated controls at 2 and $24 \mathrm{~h}$ (Fig. S1). Eighteen additional significantly dysregulated metabolites in the Ag-treatments were identified by multivariate analysis (ANOVA, $p<0.05$, Table S1). The abundances of these 45 responsive metabolites were significantly different between unexposed controls and Ag-treatments for both exposure times. The heat map clustering confirmed a sample grouping of the untreated controls and Ag treatments at 2 and $24 \mathrm{~h}$ (Fig. 4C). Five clusters were obtained, corresponding to metabolic perturbation due to Ag-treatments and time progression: Cluster 1 represents the 17 metabolites depleted under the Ag-treatments at 2 and $24 \mathrm{~h}$; Cluster 2 corresponds to 10 metabolites accumulated during the Ag-treatments, to a larger extent at $24 \mathrm{~h}$ than at $2 \mathrm{~h}$-exposure; Cluster 3 included 4 metabolites accumulated only at $24 \mathrm{~h}$; Cluster 4 

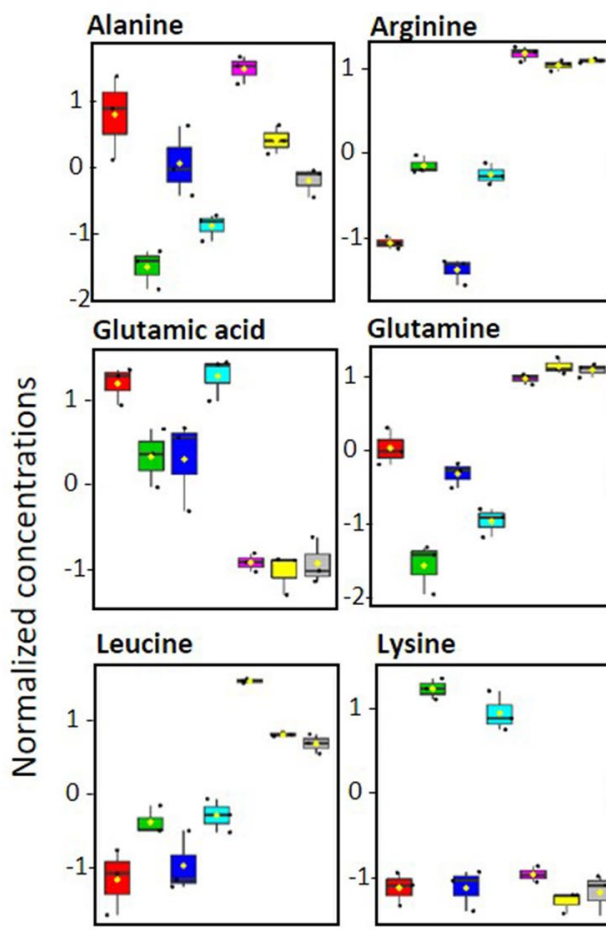

Glutamine

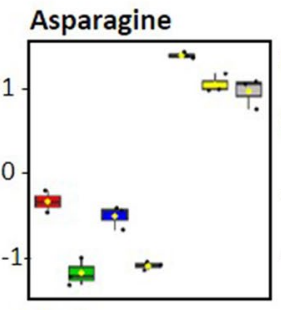

Aspartic acid

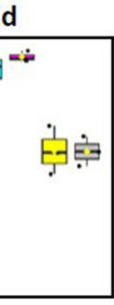

Citrulline

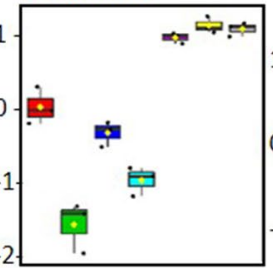

Glycine

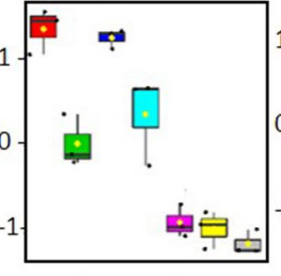

Lysine Methionine
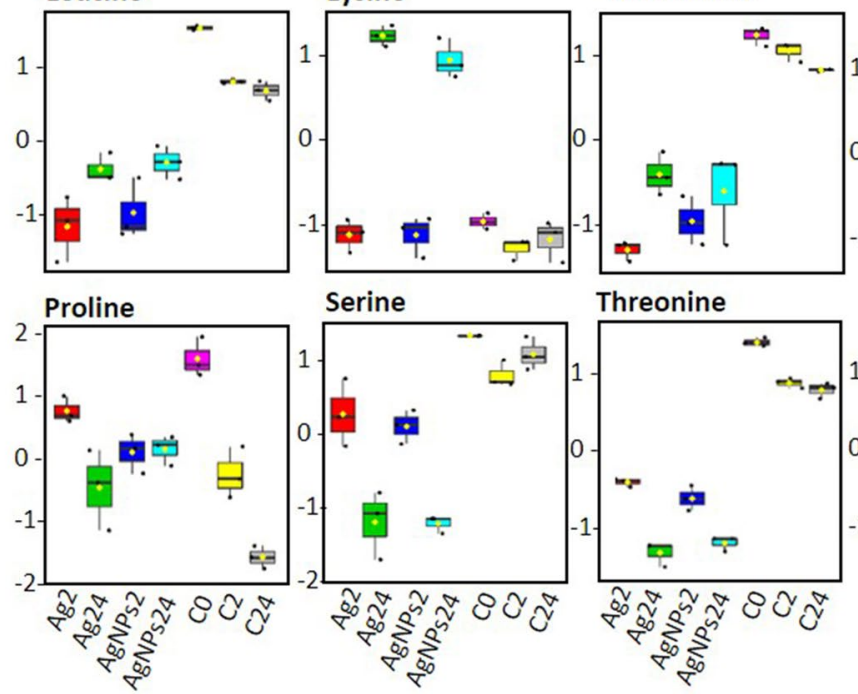

Serine

Threonine
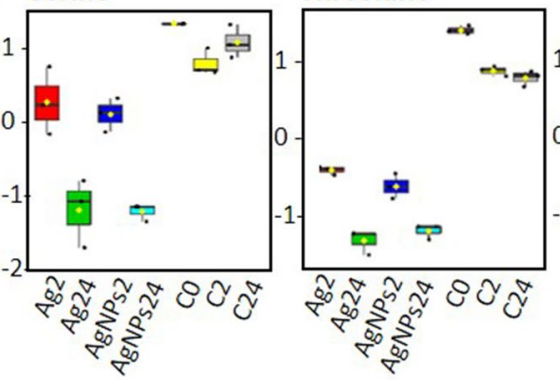

Tryptophan

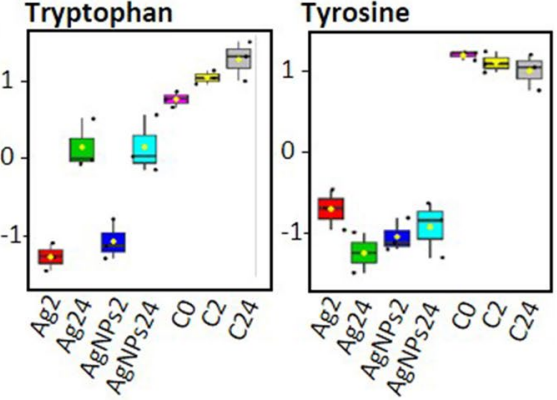

Figure 5. Box plots of relative abundance of amino acids in P. malhamensis in Ag-treatments and untreated controls. Data were normalized by using probabilistic quotient normalization by untreated control group at time 0 (C0), $\log$ transformed and autoscaled. Exposure to $40.7 \mu \mathrm{g} \mathrm{L}^{-1} \mathrm{AgNO}_{3}$ for $2 \mathrm{~h}(\mathrm{Ag} 2)$ and $24 \mathrm{~h}(\mathrm{Ag} 24)$; exposure to $1 \mathrm{mg} \mathrm{L}^{-1} \mathrm{AgNPs}$ for $2 \mathrm{~h}$ (AgNPs2) and for 24 (AgNPs24); C2: unexposed controls at time $2 \mathrm{~h}$; C24: unexposed controls at time $24 \mathrm{~h}$.

comprises 7 metabolites accumulated only at $2 \mathrm{~h}$-exposure. A more complex pattern was observed for metabolites in Cluster 5.

Time course of metabolic changes induced by AgNPs and dissolved Ag. Amino acid metabolism. Exposure of P. malhamensis to AgNPs or dissolved Ag resulted in a significant alteration of amino acid metabolism (Figs. 5, 6). Amino acids are important primary metabolites, which are the structural units of the proteins and polypeptides and serve as precursors for the synthesis of other metabolites with multiple functions in algal growth and other biological processes ${ }^{46,47}$.

A significant decrease $(p<0.05)$ in the relative abundance of a number of amino acids (arginine, asparagine, glutamine, histidine, leucine, phenylalanine, serine, threonine, tryptophan and tyrosine) was found in both $2 \mathrm{~h}$ and $24 \mathrm{~h}$ treatments (Fig. 5), suggesting either a reduction of amino acid synthesis or intensified use of these amino acids in response to Ag-treatment induced stress.

Glutamine and asparagine, two $\mathrm{N}$-rich amino acids involved in nitrate ${ }^{48}$ and ammonium ${ }^{49}$ assimilation in plants were depleted, suggesting that the capacity to acquire nitrogen compounds is lowered. Leucine, a branched chain amino acid serving as an oxidative phosphorylation energy source or as a detoxification pathway ${ }^{50}$ was also significantly decreased $(p<0.05)$ as compared with unexposed controls. Arginine and histidine, positively charged amino acids, were also significantly depleted. Histidine is an amino acid needed for growth and development of algal cells, therefore such depletion revealed the influence of both Ag-treatments on algal growth. In addition, histidine and arginine participate in deamination ${ }^{47,51}$, showing that this process can be also altered. By contrast, the abundance of aspartate, citrulline, glutamic acid, glycine, homoserine, lysine and ornithine increased with both Ag-treatments at 2 and $24 \mathrm{~h}$, suggesting an active defense of P. malhamensis against the stress induced by 


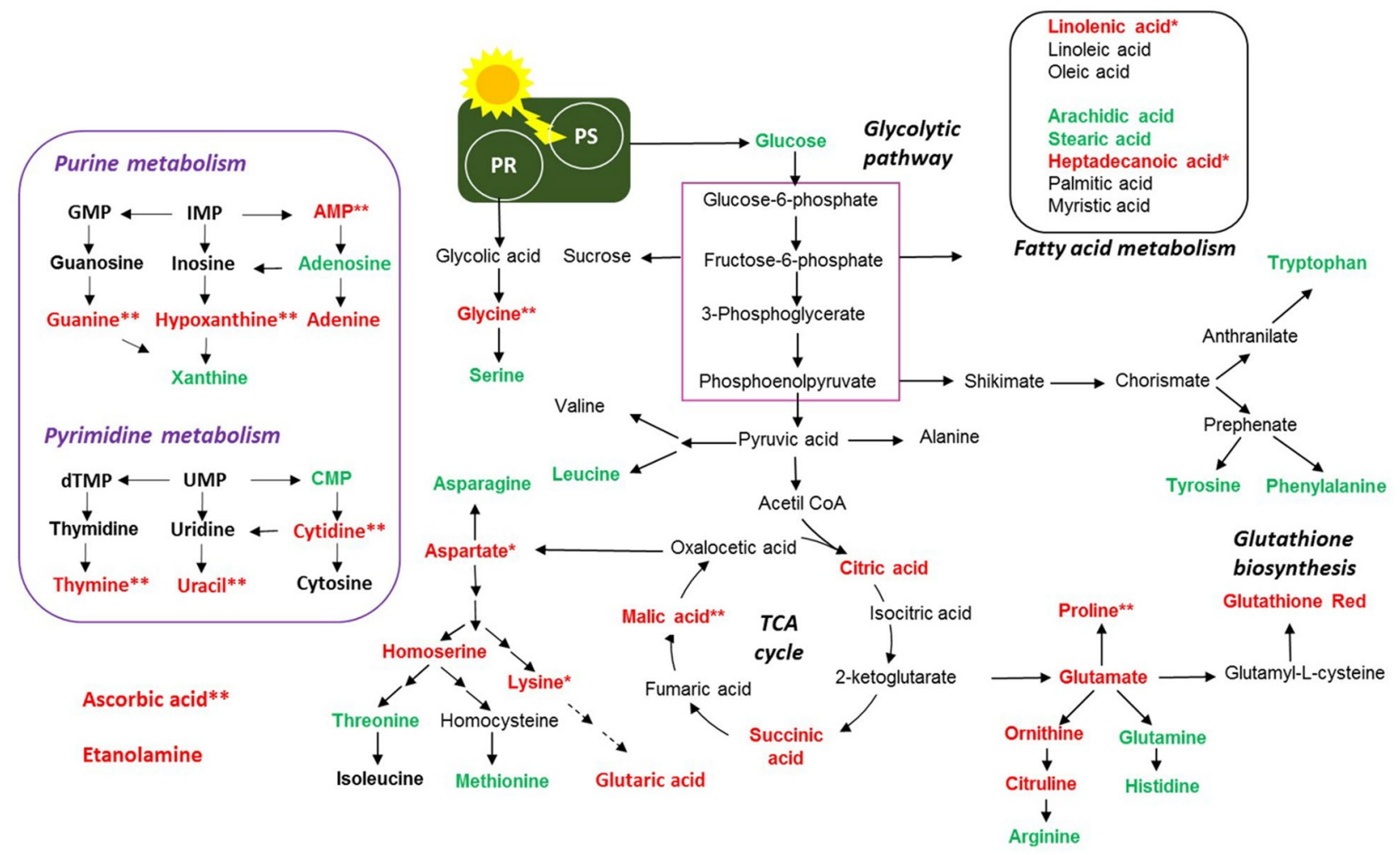

Figure 6. Overview of the proposed perturbations in metabolic pathways in golden-brown alga P. malhamensis exposed to $1 \mathrm{mg} \mathrm{L}^{-1} \mathrm{AgNPs}$ and $40.7 \mu \mathrm{g} \mathrm{L} \mathrm{AgNO}_{3}$, corresponding to the dissolved $\mathrm{Ag}$ in AgNPs suspension, for 2 and $24 \mathrm{~h}$. Accumulated and depleted metabolites are present in red and green colors. ${ }^{\star}$ denotes perturbations observed at $24 \mathrm{~h}$-exposure only; ${ }^{* *}$ denotes perturbations observed at $2 \mathrm{~h}$-exposure only, $P S$ photosynthesis, $P R$ photorespiration. Only significantly altered metabolites (Supplementary Table S1; Supplementary Fig. S1) were considered. Data were normalized by using probabilistic quotient normalization by untreated control group at time $0(\mathrm{C} 0), \log$ transformed and autoscaled.

Ag-treatments. For example, glutamic acid is known to serve as a signaling molecule and to play a role in the antioxidant defense in plants ${ }^{52}$. Moreover, the increase of glutamic acid could be related to the detoxication mechanism via induction of synthesis of phytochelatins (PCs), which form stable complexes with metals ${ }^{53}$.

Glycine and serine are part of the photorespiratory glycolate cycle in algae and their ratio is used as an indicator of the photorespiratory activity ${ }^{54}$. The Gly/Ser ratio is significantly increased in the treatments from $1.88 \pm$ 0.14 for the controls to $4.81 \pm 0.38$ and $5.32 \pm 0.52$ for $24 \mathrm{~h}$ exposures to dissolved Ag and AgNPs, respectively. Interesting, the Gly/Ser ratio also increased with exposure time from 2 to $24 \mathrm{~h}$, for example from $4.61 \pm 0.35$ to $5.32 \pm 0.52$ for AgNPs $(p<0.05)$. The change from $4.52 \pm 0.32$ to $4.81 \pm 0.38$ for $\mathrm{AgNO}_{3}$ was not statistically significant. For the unexposed controls, this ratio was unchanged over time. These results clearly demonstrated that Ag-treatments induced an increase in photorespiration, probably to produce the energy necessary for the synthesis of different defense components needed to cope with the oxidative stress in a time dependent manner. Photorespiration may provide additional protection against oxidative damage supplying glycine (also enhanced) used for synthesis of the antioxidant glutathione ${ }^{53}$. In fact, photorespiration can serve as an "energy sink, preventing the over-reduction of photosynthetic electron transport chain and photoinhibition" 55 , especially under stressed conditions that lead to reduced rates of photosynthetic $\mathrm{CO}_{2}$ assimilation as observed for Ag-treatments. These findings are in line with the reduced photochemical yield and electron transport rate of PS II (Fig. 3C) that was observed at $24 \mathrm{~h}$ exposure. Furthermore, serine can be synthesized by a non-photorespiratory pathway ${ }^{56}$, which was also probably perturbed. The accumulated amino acids can also contribute to chelation of $\mathrm{Ag}^{+}$inside the cells $s^{52}$. Methionine was significantly decreased by both Ag-treatments. Methionine is a sulfur-containing amino acid, central in many processes of the cellular metabolism, including protein synthesis ${ }^{57,58}$. Methionine is the first amino acid to be translated in protein synthesis by initiating mRNA translation and is the precursor of essential bio-molecules through S-adenosylmethionine ${ }^{59}$. It can be used to provide an estimate of the rate of protein synthesis ${ }^{60}$. Therefore, the depletion of this metabolite suggests a significant reduction of the rate of protein synthesis already at $2 \mathrm{~h}$ exposure. This is very important, because it demonstrates how metabolomics can serve to track effects well before they are observed physiologically. Given the high affinity of $\mathrm{Ag}^{+}$to $\mathrm{SH}$-groups, methionine depletion could be also due to its consumption to chelate $\mathrm{Ag}^{+}$.

Proline was significantly accumulated after only $2 \mathrm{~h}$ exposure to both Ag-treatments. Proline is known to play an important role in osmo- and redox-regulation, $\mathrm{Cd}$ and $\mathrm{Zn}$ chelation, and scavenging of free radicals induced as a result of $\mathrm{Cd}, \mathrm{Cu}, \mathrm{Hg}$ and $\mathrm{Zn}$ exposure in plants ${ }^{52,60}$. Hence, it can be hypothesized that the accumulation of proline upon exposure to AgNPs and dissolved Ag could serve as one of the first defense lines to cope with 
Ag-induced oxidative stress at $2 \mathrm{~h}$ exposure. Proline accumulation could be a result of an accelerated synthesis, release from macromolecules or inhibition of its degradation, therefore one or more of these processes could be affected by the Ag-treatments ${ }^{52}$.

Overall, significant changes in the amino acid levels with Ag treatments and over time were found, suggesting a perturbation of $\mathrm{N}$-metabolism. In addition, an $\mathrm{SH}$ - containing amino acid, methionine, is a component of S-metabolism in algae ${ }^{62}$ and its depletion suggested that $\mathrm{S}$-assimilation is disturbed.

Nucleobase/tide/side metabolism. Exposure to AgNPs and dissolved Ag altered the nucleic acids metabolism of P. malhamensis (Fig. 6, Supplementary Fig. S2). The metabolism of both purine and pyrimidine derivatives was affected. For example, a significant depletion of cytidine monophosphate (CMP), a cytosine-based nucleotide was accompanied by an accumulation of cytidine, suggesting a perturbation of the pyrimidine metabolism ${ }^{63,64}$. Similarly, guanine, hypoxanthine and adenine were accumulated in both Ag-treatments, indicating that purine metabolism was also altered ${ }^{63,64}$. Such nucleobase accumulation could be related to a nucleoside degradation due to the Ag-treatments. Nevertheless, no significant changes in the abundance of other nucleobases/tides/sides such as guanosine, inosine, thymidine, thymine, uridine and uracil were observed.

Sugars metabolism. Glucose is a primary product of photosynthesis, and depletion of glucose revealed that photosynthesis could be decreased (Fig. 6) and the alga capability to fix $\mathrm{C}$ was reduced in response to AgNPs and dissolved Ag. Photosynthesis is a fundamental process, converting $\mathrm{CO}_{2}$ to organic carbon available to phytoplankton cells. The decrease of the abundance of glucose upon Ag-treatments was more pronounced in AgNPs treatment and at $24 \mathrm{~h}$ exposure. The above finding is consistent with the observed inhibition of the photochemical yield at $24 \mathrm{~h}$ exposure (Fig. 3C) and the two-fold increase of NPQ by AgNPs and dissolved Ag (Fig. 3D). This observation agrees with the accelerated photorespiration observed during both Ag-treatments. In addition, a decrease in glucose concentration and the increase of TCA metabolites could also be related with induction of the mitochondrial activity ${ }^{65}$.

Fatty acids metabolism. The unsaturated fatty acid linolenic acid (C 9,12,15 double bonds) accumulated more after 24 h exposure to AgNPs and dissolved Ag, while linoleic (C 9,12 double bonds) and oleic (C 9 double bond) acids were unchanged. In parallel, a significant depletion of the saturated acids-arachidic (C20 strait chain), stearic (C18 strait chain) acids and to a lower degree heptadecanoic acid (C17 strait chain) - was observed (Fig. 6, Supplementary Fig. S3). Other saturated fatty acids, such as palmitic acid (C16 strait chain), were unaffected. These metabolite changes indicate that Ag-treatment induced unsaturation of the lipid membranes and altered the composition and integrity of lipid membranes. Indeed, this finding agrees with the strong lipid peroxidation observed in both Ag-treatments (Fig. 3B). A perturbation of the metabolism of fatty acids can also change the energy budget ${ }^{66}$. An alteration of the fatty acid composition is a common observation under stress, and algae have been shown to adjust the degree of unsaturation of the membrane to combat oxidative stress induced by toxic metals ${ }^{67}$. Furthermore, plants could adjust the composition of fatty acids in the membrane to rebuild membrane integrity, as was shown for cucumber leaves exposed to AgNPs and dissolved $\mathrm{Ag}^{5}$. Treatments with AgNPs and dissolved Ag were also shown to reduce monounsaturated and polyunsaturated fatty acids of green microalgae Chlorella vulgaris ${ }^{68}$.

Carboxylic acid metabolism and antioxidants. Citric acid and succinic acid, intermediates in the tricarboxylic acid (TCA) cycle significantly accumulated in both Ag-treatments after $2 \mathrm{~h}$ and $24 \mathrm{~h}$ (Fig. 6, Supplementary Fig. S4). With the exception of the $2 \mathrm{~h}$ AgNPs treatment, malic acid levels in $P$. malamensis increased significantly $(p<0.05)$ in all other treatments. This finding clearly showed that the TCA cycle, which is key metabolic pathway that connects carbohydrate, fatty acids, and protein metabolism, was accelerated. The TCA cycle is the core of the cell's respiratory machinery; it is likely that algae accelerate respiration to produce energy necessary for the manufacture of defense compounds needed to address oxidative stress. This observation is consistent with the increased ratios of the Gly/Ser (see "Amino acid metabolism"). Exposure to AgNPs and dissolved Ag led to the significant increase in the concentrations of two antioxidant molecules: ascorbic acid and reduced glutathione (GSH) (Fig. 6). The accumulation of the ascorbic acid was more pronounced at $2 \mathrm{~h}$ exposure than in $24 \mathrm{~h}$, while the increase in the GSH was greater in $24 \mathrm{~h}$ treatments. The results clearly indicate that the antioxidant defense system of $P$. malamensis was activated by Ag-treatments, which resulted in an accumulation of the ROS-scavenging metabolites to cope with the enhanced generation of ROS (Fig. 3A). In addition, GSH is a precursor of PCs which is activated by different toxic metals including $\mathrm{Ag}^{69}$.

Taken together, the decrease of fatty acids, the increase of the antioxidants level, such as GSH, and GSH-related amino acids glycine and glutamate, suggest that the AgNPs and dissolved Ag triggered an excessive generation of ROS, in agreement with experimentally observed oxidative stress (Fig. 3A) and lipid peroxidation (Fig. 3B). These observations corroborate the significant accumulation of glutaric acid after Ag-treatments, since glutaric acid, is a product of the fatty acid and lysine degradation metabolism.

Metabolic response specific to AgNPs. To discriminate between metabolic perturbations induced by AgNPs and dissolved Ag, separate analyses of the respective data sets for $2 \mathrm{~h}$ and $24 \mathrm{~h}$ were conducted (Supplementary Fig. S5, Supplementary Table S2; Supplementary Fig. S6, Supplementary Table S3). For both $2 \mathrm{~h}$ an $24 \mathrm{~h}$ exposures, the PCA score plot (Fig. S5A, S6A) confirmed good separation between AgNPs, dissolved Ag and unexposed controls. A supervised PLS-DA score plot (Supplementary Figs. S5B, S6B) resulted in clear separation between exposures to AgNPs and dissolved Ag. The responsive metabolites, isolated on the basis of VIP score $>1$ included 33 molecules for 2 h exposure (Supplementary Fig. S5C) and 34 molecules for 24 h exposure 
(Supplementary Fig. S6C). Metabolites commonly or specifically perturbed at $2 \mathrm{~h}$ and 24 h-exposure which discriminate AgNPs and dissolved Ag responses are present in Supplementary Fig. S7.

No specific metabolites were affected in response to AgNPs only. However, some metabolites were either accumulated or depleted to a larger extent after exposure to AgNPs as compared with dissolved Ag. As the experiment was designed to distinguish the responses in the presence of $\mathrm{AgNPs}$ and $\mathrm{AgNO}_{3}$ corresponding to the dissolved $\mathrm{Ag}$ in the particle suspensions, these metabolic changes showed an important contribution of the particulate form to the overall response. At $2 \mathrm{~h}$ exposure to AgNPs adenine, glycine and homoserine were upregulated more strongly $(p<0.05)$ than in dissolved Ag-exposure; while arginine, asparagine, glutamine, histidine, threonine, tyrosine and xanthine were depleted to a larger extent $(p<0.05)$, indicating the important contribution of AgNPs to the perturbation of amino acid metabolism. CMP was depleted, while cytidine abundance increased more strongly in AgNPs than in the dissolved Ag-treatments, showing significant involvement of AgNPs in the perturbation of pyrimidine metabolism. A higher accumulation of GSH was also found in AgNPs treatments at $2 \mathrm{~h}$, pointing out the important role of AgNPs in induction of oxidative stress, which corroborate the results of the cellular ROS generation (Fig. 3A). A bigger accumulation of succinic acid in AgNPs than in dissolved Agtreatment indicated a significant role of the AgNPs in TCA cycle perturbation. Glucose levels decreased more after $2 \mathrm{~h}$ exposure to AgNPs, in agreement with the photosynthesis inhibition results. Arachidic acid depletion was also larger in AgNPs than dissolved Ag-treatments (Supplementary Fig. S5). At 24 h exposure to AgNPs arginine and methionine decrease was stronger; whereas aspartic acid, citrulline, glycine, glutamic acid, glutaric acid, homoserine, ornithine, proline increase was more pronounced. Adenine and AMP were accumulated to a larger extent, suggesting stronger impact of the AgNPs on the purine metabolism (Supplementary Fig. S6). At $24 \mathrm{~h}$ exposure, no significant difference was found in the AgNPs and dissolved Ag-induced perturbation in the TCA cycle, fatty acid metabolism and GSH concentrations.

\section{Conclusion}

The present study for the first time revealed the metabolic perturbations induced by AgNPs following their uptake and accumulation in the food vacuoles of golden-brown alga P. malhamensis. Results of targeted metabolomics demonstrated that the exposure to AgNPs and dissolved Ag resulted in time-dependent perturbation of the concentration of metabolites involved in various metabolic pathways involving amino acids, nucleotides, fatty acids, TCA, antioxidants, photosynthesis and photorespiration. Even though the intensity of the responses differed with exposure time and type of Ag in the treatments, the observed metabolic perturbations were common for the particulate and dissolved forms. The results suggest that dissolved Ag released by AgNPs are the major toxicity driver, even though AgNPs are internalized in the food vacuoles. However, AgNPs play an important role in the perturbation of amino acid metabolism, TCA cycle and oxidative stress, in particularly at $2 \mathrm{~h}$ exposure. Their role diminished at $24 \mathrm{~h}$ since the AgNPs in the food vacuoles aggregated substantially by then, and thus released less $\mathrm{Ag}^{+}$. The metabolomic perturbations were reflected in the physiological responses such as increased cellular ROS generation (more pronounced in AgNPs, treatments); lipid peroxidation in P. malhamensis, and decreases of the photosynthetic efficiency for both Ag-treatments with time. This study demonstrates the value of metabolomics as a tool for understanding the molecular basis for these metabolic and physiological changes, and to detect early on metabolic changes that can later express themselves physiologically.

\section{Methods}

Characterization of AgNPs in exposure medium. Citrate-coated spherical AgNPs (BioPure) with a primary size of $20 \mathrm{~nm}$ at $1.01 \mathrm{~g} \mathrm{Ag} \mathrm{L}^{-1}\left(2 \times 10^{13} \mathrm{NP} \mathrm{mL}^{-1}\right)$ in $2 \mathrm{mM}$ citrate, were purchased from Nanocomposix (San Diego, USA). Silver nitrate $\left(\mathrm{AgNO}_{3}\right.$ ) were purchased from Sigma-Aldrich. The characteristics of the AgNPs in the stock suspension as provided by the manufacturer can be found in Supplementary Fig. S8. AgNP suspensions in the exposure medium was characterized in terms of dissolution and aggregation at $2 \mathrm{~h}$ and $24 \mathrm{~h}$. The hydrodynamic diameters and their distributions, and the zeta potential (ZP) were measured using a Malvern Zetasizer Nano (Malvern Instruments Inc, UK). The size and morphology of the AgNPs in the exposure medium were determined by transmission electronic microscopy (TEM) (FEI Tecnai ${ }^{\text {Th }}$ G2 Sphera, FEI Company, USA). The percentage of dissolved silver in the suspensions of AgNPs at 2 and $24 \mathrm{~h}$ was assessed by ultracentrifugation and analysis of the dissolved Ag concentration in the supernatant by inductively coupled plasma mass spectrometry (ICP-MS, Agilent 7700x, Basel, Switzerland) as detailed in the SI.

Bioassays with P. malhamensis. Poterioochromonas malhamensis (CCAC 3498 strain, Cologne Biocenter, Germany) was grown in a modified Waris- $\mathrm{H}$ medium (Supplementary Table S4) at $25{ }^{\circ} \mathrm{C}$ with a light illumination of $5 \mu \mathrm{mol}$ photons $\mathrm{m}^{-2} \mathrm{~s}^{-1}$ in a 12:12 light-dark cycle in the specialized incubator (MIR 253, Sanyo, Japan). The cells were harvested and re-suspended in the modified Waris-H medium enriched with AgNPs to a cell density of $10^{6}$ cells $\mathrm{mL}^{-1}$. The suspension of $1 \mathrm{mgL}^{-1}$ AgNPs in the exposure medium were prepared by 1000 times dilution of the stock suspension $\left(\mathrm{g} \mathrm{L}^{-1}\right)$ and homogenization by vortex for $45 \mathrm{~s}$ before use. Uptake, physiological and metabolic perturbations were determined at 2 and $24 \mathrm{~h}$. To distinguish between the effects of AgNPs and dissolved Ag present in the AgNPs suspensions on the algal metabolism and physiological effects, the experiments were performed in the presence of $1 \mathrm{mg} \mathrm{L}^{-1} \mathrm{AgNPs}$ and $40.7 \mu \mathrm{g} \mathrm{L}^{-1} \mathrm{AgNO}_{3}$ at 2 and $24 \mathrm{~h}$-exposure. The concentration of $\mathrm{AgNO}_{3}$ corresponds to the dissolved $\mathrm{Ag}$ in the suspensions of $1 \mathrm{mgL}^{-1} \mathrm{AgNPs}$. The choice of AgNPs concentrations was made based on some preliminary bioassays (Supplementary Figs. S9-S12).

Ag cellular burden and AgNPs internalization. To measure the total Ag burden upon AgNPs and $\mathrm{AgNO}_{3}$ exposure, the exposed cell suspensions were centrifuged at $1000 \mathrm{~g}$ for $10 \mathrm{~min}$. The pellets containing algae were rinsed twice with Ag free Waris- $\mathrm{H}$ medium to remove all loosely bound particles from algal surface 
and then digested in ultrapure $\mathrm{HNO}_{3}$ overnight at $90{ }^{\circ} \mathrm{C}$ in an oven, diluted with MilliQ water and analyzed by ICP-MS (Agilent 7700x, Basel, Switzerland).

To determine the cellular distribution of AgNPs in P. melhamensis, the cells were fixed with $2 \%$ glutaraldehyde and $4 \%$ formaldehyde overnight at $4{ }^{\circ} \mathrm{C}$. After post-fixation staining with $1 \%$ osmium tetroxide, the cells were embedded in epoxy resin. Ultrathin section $(80 \mathrm{~nm}$ ) were obtained with an ultramicrotome (Leica, Bannockburn, IL, USA). These sections were stained with $2 \%$ uranyl acetate solution and plumbic citrate, and then placed on 200 mesh copper grids. Morphological characteristics of cells and the distribution of particles within the cells were analyzed with Transmission Electron Microscopy (TEM, FEI Tecnai ${ }^{\text {tw }}$ G2 Sphera, FEI Company, USA) operated at $80 \mathrm{kV}$. Energy dispersive X-ray detector (AMETEK Inc. Germany) was used to carry out the chemical analysis. The measurement and analyses were operated by the software Genesis version 6.255.

Physiological effects of AgNPs and $\mathrm{AgNO}_{3}$. The influence of $\mathrm{AgNPs}$ and $\mathrm{AgNO}_{3}$ on cell growth, generation of ROS, lipid peroxidation and photosynthetic activity was assessed as detailed in the SI. Briefly, cellular ROS generation was followed by BD Accuri C6 flow cytometer (BD Biosciences, San Jose, CA) using the CellROX@Green stain (Life Technologies Europe B.V., Zug, Switzerland) following the procedure adapted from ${ }^{70}$. Unexposed algae were used as negative control, while algae exposed to $2.5 \mathrm{mM} \mathrm{H}_{2} \mathrm{O}_{2}$ for 20 min were used as positive control. Lipid peroxidation was assessed by malondialdehyde (MAD) kit (Sigma-Aldrich. St Louis, USA) and spectrophotometry detection. Changes in the photosynthetic activity of $P$. malhamensis during Agtreatments were followed using a Multiple Excitation Wavelength Chlorophyll Fluorescence Analyzer (MultiColor-PAM, Walz, Germany). Maximal fluorescence yield of the photosystem II, Fm, and maximal variable fluorescence, $\mathrm{Fv}(\mathrm{Fv} / \mathrm{Fm})$ and non-photochemical quenching (NPQ), corresponding to the dissipation of excess energy as heat loss from PSII, were measured after $2 \mathrm{~h}$ and $24 \mathrm{~h}$ exposure following 20 min of dark acclimation. These parameters are well-known indicators for alteration of photosynthetic activity by different biotic and abiotic stressors ${ }^{71}$. Results are present in Supplementary Fig. S12.

Statistically significant differences of the percentage of affected cells by $\mathrm{AgNPs}$ and $\mathrm{AgNO}_{3}$ were analyzed using a two-way analysis of variance (ANOVA) followed by a Sidak's multiple comparisons test (Graphpad Prism 6, Graphpad Sofeware Inc., San Diego, CA, USA).

LC-MS targeted metabolomics. Metabolic changes in P. malhamensis exposed to $1 \mathrm{mg} \mathrm{L}^{-1}$ AgNPs or $40.7 \mu \mathrm{g} \mathrm{L}^{-1} \mathrm{AgNO}_{3}$ for 2 and $24 \mathrm{~h}$ were determined by LC-MS targeted metabolomics. Untreated algae were used as control. Control and exposed $P$. malhamensis were sampled at $0 \mathrm{~h}$ ( $\mathrm{t} 0$, beginning of the exposure), $2 \mathrm{~h}$ and $24 \mathrm{~h}$ to assess the variation in metabolite levels during the cell development cycle. At the end of the exposure, the cells were put in liquid nitrogen to stop metabolic activity, then frozen at $-80^{\circ} \mathrm{C}$ for $24 \mathrm{~h}$ and freeze-dried. Different metabolites, including antioxidants, amines, amino acids, organic acids/phenolics, nucleobase/side/ tide, sugar/sugar alcohols and fatty acids were extracted in $80 \%$ methanol containing $2 \%$ formic acid following previously developed methodology ${ }^{8,9}$. Targeted analyses of the metabolites were performed using Agilent 6470 liquid chromatography triple quadrupole mass spectrometer according to previously established methods with MS parameters, as shown in Supplementary Table $\mathrm{S}^{4,8,9}$.

Statistical analysis of the metabolomics data was performed by using MetaboAnalyst $4.0^{72}$. Data were normalized using the probabilistic quotient normalization by the unexposed control group at 0 , then log-normalized and autoscaled. Exploratory data analysis was performed with one-way analysis of variance (ANOVA) followed by a Fisher's least significant difference method (Fisher's LSD) with $p$ value threshold of 0.05 . Unsupervised Principal Component Analysis (PCA) and supervised Partial Least Squares-Discriminant Analysis (PLS-DA) were employed to cluster different treatments. Variables with a importance in the projection (VIP) greater than 1 were regarded as significant and responsible for group separation discriminating metabolites ${ }^{73}$. The changes in the abundance of measured metabolites are illustrated in the boxplots, generated by MetaboAnalyst 4.0. The plots were treated to increase the level size and axis titles and thus improve the readability.

\section{Data availability}

All data supporting the findings of this study are available within the article and its supplementary information file.

Received: 22 April 2020; Accepted: 12 November 2020

Published online: 25 November 2020

\section{References}

1. Fiehn, O. Metabolomics-the link between genotypes and phenotypes. Plant Mol. Biol. 48, 155-171. https://doi.org/10.1023/a:10137 13905833 (2002)

2. Volz, D. C. et al. Adverse outcome pathways during early fish development: A conceptual framework for identification of chemical screening and prioritization strategies. Toxicol. Sci. 123, 349-358 (2011).

3. Bundy, J. G., Davey, M. P. \& Viant, M. R. Environmental metabolomics: A critical review and future perspectives. Metabolomics 5, 3. https://doi.org/10.1007/s11306-008-0152-0 (2008).

4. Majumdar, S. et al. Proteomic, gene and metabolite characterization reveal the uptake and toxicity mechanisms of cadmium sulfide quantum dots in soybean plants. Environ. Sci. Nano 6, 3010-3026. https://doi.org/10.1039/c9en00599d (2019).

5. Zhang, H. L. et al. Metabolomics reveals how cucumber (Cucumis sativus) reprograms metabolites to cope with silver ions and silver nanoparticle-induced oxidative stress. Environ. Sci. Technol. 52, 8016-8026. https://doi.org/10.1021/acs.est.8b02440 (2018).

6. Zhao, L. J., Huang, Y. X., Adeleye, A. S. \& Keller, A. A. Metabolomics reveals $\mathrm{Cu}(\mathrm{OH})_{2}$ nanopesticide-activated anti-oxidative pathways and decreased beneficial antioxidants in spinach leaves. Environ. Sci. Technol. 51, 10184-10194. https://doi.org/10.1021/ acs.est.7b02163 (2017). 
7. Zhao, L. J., Huang, Y. X. \& Keller, A. A. Comparative metabolic response between Cucumber (Cucumis sativus) and Corn (Zea mays) to a $\mathrm{Cu}(\mathrm{OH})_{2}$ nanopesticide. J. Agric. Food Chem. 66, 6628-6636. https://doi.org/10.1021/acs.jafc.7b01306 (2018).

8. Huang, Y. et al. Antioxidant response of cucumber (Cucumis sativus) exposed to nano copper pesticide: Quantitative determination via LC-MS/MS. Food Chem. 270, 47-52. https://doi.org/10.1016/j.foodchem.2018.07.069 (2019).

9. Huang, Y., Li, W., Minakova, A. S., Anumol, T. \& Keller, A. A. Quantitative analysis of changes in amino acids levels for cucumber (Cucumis sativus) exposed to nano copper. NanoImpact 12, 9-17. https://doi.org/10.1016/j.impact.2018.08.008 (2018).

10. Matich, E. K., Soria, N. G. C., Aga, D. S. \& Atilla-Gokcumen, G. E. Applications of metabolomics in assessing ecological effects of emerging contaminants and pollutants on plants. J. Hazard. Mater. 373, 527-535. https://doi.org/10.1016/j.jhazmat.2019.02.084 (2019).

11. Li, X. K., Peng, T., Mu, L. \& Hu, X. G. Phytotoxicity induced by engineered nanomaterials as explored by metabolomics: Perspectives and challenges. Ecotoxicol. Environ. Saf. 184, 10. https://doi.org/10.1016/j.ecoenv.2019.109602 (2019).

12. Zhang, J. L. et al. Metabolic profiling of silver nanoparticle toxicity in Microcystis aeruginosa. Environ. Sci. Nano 5, 2519-2530. https://doi.org/10.1039/c8en00738a (2018).

13. Falkowski, P. Ocean science: The power of plankton. Nature 483, S17-S20. https://doi.org/10.1038/483S17a (2012).

14. McGillicuddy, E. et al. Silver nanoparticles in the environment: Sources, detection and ecotoxicology. Sci. Total Environ. 575, 231-246. https://doi.org/10.1016/j.scitotenv.2016.10.041 (2017).

15. Cervantes-Avilés, P., Huang, Y. \& Keller, A. A. Incidence and persistence of silver nanoparticles throughout the wastewater treatment process. Water Res. 156, 188-198. https://doi.org/10.1016/j.watres.2019.03.031 (2019).

16. Zhang, W., Xiao, B. \& Fang, T. Chemical transformation of silver nanoparticles in aquatic environments: Mechanism, morphology and toxicity. Chemosphere 191, 324-334. https://doi.org/10.1016/j.chemosphere.2017.10.016 (2018).

17. Boenigk, J. et al. Effects of silver nitrate and silver nanoparticles on a planktonic community: General trends after short-term exposure. J. PloS One 9, e95340 (2014).

18. Kalantzi, I. et al. Ecotoxicity of silver nanoparticles on plankton organisms: A review. J. Nanopart. Res. 21, 65 (2019).

19. Domingo, G., Bracale, M. \& Vannini, C. Nanomaterials in Plants, Algae and Microorganisms 143-168 (Elsevier, New York, 2019).

20. Slaveykova, V. I., Li, M., Worms, I. A. \& Liu, W. When environmental chemistry meets ecotoxicology: Bioavailability of inorganic nanoparticles to phytoplankton. CHIMIA Int. J. Chem. 74, 115-121. https://doi.org/10.2533/chimia.2020.115 (2020).

21. Ivask, A. et al. Mechanisms of toxic action of $\mathrm{Ag}, \mathrm{ZnO}$ and $\mathrm{CuO}$ nanoparticles to selected ecotoxicological test organisms and mammalian cells in vitro: A comparative review. Nanotoxicology 8, 57-71. https://doi.org/10.3109/17435390.2013.855831 (2014).

22. von Moos, N. \& Slaveykova, V. I. Oxidative stress induced by inorganic nanoparticles in bacteria and aquatic microalgae-state of the art and knowledge gaps. Nanotoxicology 8, 605-630. https://doi.org/10.3109/17435390.2013.809810 (2014).

23. Bock, C. et al. Silver stress differentially affects growth of phototrophic and heterotrophic chrysomonad flagellate populations. Environ. Pollut. 244, 314-322. https://doi.org/10.1016/j.envpol.2018.09.146 (2019).

24. Ribeiro, F. et al. Uptake and elimination kinetics of silver nanoparticles and silver nitrate by Raphidocelis subcapitata: The influence of silver behaviour in solution. Nanotoxicology 9, 686-695. https://doi.org/10.3109/17435390.2014.963724 (2015).

25. Piccapietra, F., Allue, C. G., Sigg, L. \& Behra, R. Intracellular silver accumulation in Chlamydomonas reinhardtii upon exposure to carbonate coated silver nanoparticles and silver nitrate. Environ. Sci. Technol. 46, 7390-7397. https://doi.org/10.1021/es300734m (2012)

26. Miao, A. J. et al. Intracellular uptake: A possible mechanism for silver engineered nanoparticle toxicity to a freshwater alga Ochromonas danica. PLoS One 5, e15196. https://doi.org/10.1371/journal.pone.0015196 (2010).

27. Wang, S., Lv, J., Ma, J. \& Zhang, S. Cellular internalization and intracellular biotransformation of silver nanoparticles in Chlamydomonas reinhardtii. Nanotoxicology 10, 1129-1135. https://doi.org/10.1080/17435390.2016.1179809 (2016).

28. Stoecker, D. K. Conceptual models of mixotrophy in planktonic protists and some ecological and evolutionary implications. Eur. J. Protistol. 34, 281-290 (1998).

29. Zhang, X. \& Watanabe, M. M. Grazing and growth of the mixotrophic chrysomonad Poterioochromonas malhamensis (Chrysophyceae) feeding on algae. J. Phycol. 37, 738-743 (2001).

30. Sherr, E. B. \& Sherr, B. F. Significance of predation by protists in aquatic microbial food webs. Antonie Van Leeuwenhoek 81, 293-308 (2002).

31. Cole, G. T. \& Wynne, M. Endocytosis of microcystis Aeruginosa by Ochromonas danica. J. Phycol. 10, 397-410 (1974).

32. OECD. Silver Nanoprticles; Summary of the Dossier (Oeganisation for Economic Cooperation and Development, Paris, 2017).

33. Lead, J. R. et al. Nanomaterials in the environment: Behavior, fate, bioavailability, and effects. An updated review. Environ. Toxicol. Chem. 37, 2029-2063. https://doi.org/10.1002/etc.4147 (2018).

34. Yue, Y. et al. Toxicity of silver nanoparticles to a fish gill cell line: Role of medium composition. Nanotoxicology 9, 54-63. https:// doi.org/10.3109/17435390.2014.889236 (2015).

35. Li, X. et al. Silver nanoparticle toxicity and association with the alga Euglena gracilis. Environ. Sci. Nano 2, 594-602. https://doi. org/10.1039/c5en00093a (2015).

36. Sunda, W. G. \& Huntsman, S. A. Processes regulating cellular metal accumulation and physiological effects: Phytoplankton as model systems. Sci. Total Environ. 219, 165-181. https://doi.org/10.1016/S0048-9697(98)00226-5 (1998).

37. Ma, M., Gong, Y. \& Hu, Q. Identification and feeding characteristics of the mixotrophic flagellate Poterioochromonas malhamensis, a microalgal predator isolated from outdoor massive Chlorella culture. Algal Res. 29, 142-153. https://doi.org/10.1016/j.algal 2017.11.024 (2018).

38. Oukarroum, A., Bras, S., Perreault, F. \& Popovic, R. Inhibitory effects of silver nanoparticles in two green algae, Chlorella vulgaris and Dunaliella tertiolecta. Ecotoxicol. Environ. Saf. 78, 80-85 (2012).

39. Farmer, E. E. \& Mueller, M. J. ROS-mediated lipid peroxidation and RES-activated signaling. Annu. Rev. Plant Biol. 64, 429-450. https://doi.org/10.1146/annurev-arplant-050312-120132 (2013).

40. Demidchik, V. Mechanisms of oxidative stress in plants: From classical chemistry to cell biology. Environ. Exp. Bot. 109, $212-228$. https://doi.org/10.1016/j.envexpbot.2014.06.021 (2015).

41. Ritchie, R. J. Fitting light saturation curves measured using modulated fluorometry. Photosynth. Res. 96, 201-215. https://doi. org/10.1007/s11120-008-9300-7 (2008).

42. Miller, R. J. et al. Photosynthetic efficiency predicts toxic effects of metal nanomaterials in phytoplankton. Aquat. Toxicol. 183, 85-93. https://doi.org/10.1016/j.aquatox.2016.12.009 (2017).

43. Baptista, M. S. et al. Impacts of silver nanoparticles on a natural estuarine plankton community. Environ. Sci. Technol. 49, 1296812974. https://doi.org/10.1021/acs.est.5b03285 (2015).

44. Pillai, S. et al. Linking toxicity and adaptive responses across the transcriptome, proteome, and phenotype of Chlamydomonas reinhardtii exposed to silver. Proc. Natl. Acad. Sci. USA 111, 3490-3495. https://doi.org/10.1073/pnas.1319388111 (2014).

45. Latifi, A., Ruiz, M. \& Zhang, C.-C. Oxidative stress in cyanobacteria. FEMS Microbiol. Rev. 33, 258-278. https://doi.org/10.111 1/j.1574-6976.2008.00134.x (2009).

46. Bromke, M. A. Amino acid biosynthesis pathways in diatoms. Metabolites 3, 294-311 (2013).

47. Hildebrandt, T. M., Nunes, A. N., Araújo, W. L. \& Braun, H.-P. Amino acid catabolism in plants. Mol. Plant 8, 1563-1579. https:// doi.org/10.1016/j.molp.2015.09.005 (2015).

48. Joy, K. Ammonia, glutamine and asparagine: A carbon-nitrogen interface. Can. J. Bot. 66, 2103-2109 (2011). 
49. Forde, B. G. \& Lea, P. J. Glutamate in plants: Metabolism, regulation, and signaling. J. Exp. Bot. 58, 2339-2358. https://doi. org/10.1093/jxb/erm121 (2007).

50. Taylor, N. L., Heazlewood, J. L., Day, D. A. \& Millar, A. H. Lipoic acid-dependent oxidative catabolism of alpha-keto acids in mitochondria provides evidence for branched-chain amino acid catabolism in Arabidopsis. Plant Physiol. 134, 838-848. https:// doi.org/10.1104/pp.103.035675 (2004).

51. Lieberman, M. A. \& Ricer, R. Brs Biochemistry, Molecular Biology, And Genetics 6th edn. (Wolters Kluwer, Lippincott Williams and Wilkins, Philadelphia, 2013).

52. Sharma, S. S. \& Dietz, K.-J. The significance of amino acids and amino acid-derived molecules in plant responses and adaptation to heavy metal stress. J. Exp. Bot. 57, 711-726. https://doi.org/10.1093/jxb/erj073 (2006).

53. Devez, A., Achterberg, E. \& Gledhill, M. in Metallothioneins and Related Chelators 5 (eds A. Sigel, H. Sigel, \& R. K. O. Sigel) 441-481 (Royal Society of Chemistry, 2009).

54. Timm, S. et al. Serine acts as a metabolic signal for the transcriptional control of photorespiration-related genes in Arabidopsis. Plant Physiol. 162, 379-389. https://doi.org/10.1104/pp.113.215970 (2013).

55. Wingler, A., Lea, P. J., Quick, W. P. \& Leegood, R. C. Photorespiration: Metabolic pathways and their role in stress protection. Philos. Trans. R. Soc. Lond. B Biol. Sci. 355, 1517-1529. https://doi.org/10.1098/rstb.2000.0712 (2000).

56. Vallon, O. \& Spalding, M. H. The Chlamydomonas Sourcebook Vol 2 115-158 (Academic Press Inc, New York, 2009).

57. Ravanel, S., Gakière, B., Job, D. \& Douce, R. The specific features of methionine biosynthesis and metabolism in plants. Proc. Natl. Acad. Sci. 95, 7805-7812. https://doi.org/10.1073/pnas.95.13.7805 (1998).

58. Amir, R. \& Hacham, Y. in Sulfur: A Missing Link Between Soils, Crops, and Nutrition Agronomy Monographs (ed Joseph Jez) 251-279 (American Society of Agronomy, Crop Science Society of America, Soil Science Society of America, 2008).

59. Wirtz, M. \& Droux, M. Synthesis of the sulfur amino acids: Cysteine and methionine. Photosynth. Res. 86, 345-362. https://doi. org/10.1007/s11120-005-8810-9 (2006).

60. Lameka, K., Farwell, M. D. \& Ichise, M. In Handbook of Clinical Neurology*Vol 135 (eds Masdeu, J. C. \& González, R. G.) 209-227 (Elsevier, New York, 2016).

61. Matysik, J., Alia, B. B. \& Mohanty, P. Molecular mechanisms of quenching of reactive oxygen species by proline under stress in plants. Curr. Sci. 82, 525-532 (2002).

62. Giordano, M., Norici, A., Ratti, S. \& Raven, J. 397-415 (2008)

63. Stasolla, C., Katahira, R., Thorpe, T. A. \& Ashihara, H. Purine and pyrimidine nucleotide metabolism in higher plants. J. Plant Physiol. 160, 1271-1295. https://doi.org/10.1078/0176-1617-01169 (2003).

64. Moffatt, B. \& Ashihara, H. Purine and pyrimidine nucleotide synthesis and metabolism. Arabidopsis Book Am. Soc. Plant Biol. 1, e0018. https://doi.org/10.1199/tab.0018 (2002).

65. Martínez-Reyes, I. \& Chandel, N. S. Mitochondrial TCA cycle metabolites control physiology and disease. Nat. Commun. 11, 102. https://doi.org/10.1038/s41467-019-13668-3 (2020).

66. Filimonova, V., Gonçalves, F., Marques, J. C., De Troch, M. \& Gonçalves, A. M. M. Fatty acid profiling as bioindicator of chemical stress in marine organisms: A review. Ecol. Ind. 67, 657-672. https://doi.org/10.1016/j.ecolind.2016.03.044 (2016).

67. Pinto, E. et al. Heavy metal-induced oxidative stress in algae. J. Phycol. 39, 1008-1018. https://doi.org/10.1111/j.0022-3646.2003.02193.x (2003).

68. Behzadi Tayemeh, M. et al. Perturbation of fatty acid composition, pigments, and growth indices of Chlorella vulgaris in response to silver ions and nanoparticles: A new holistic understanding of hidden ecotoxicological aspect of pollutants. Chemosphere 238, 124576. https://doi.org/10.1016/j.chemosphere.2019.124576 (2020).

69. Gekeler, W., Grill, E., Winnacker, E.-L. \& Zenk, M. H. Algae sequester heavy metals via synthesis of phytochelatin complexes. Arch. Microbiol. 150, 197-202. https://doi.org/10.1007/BF00425162 (1988).

70. Cheloni, G., Marti, E. \& Slaveykova, V. I. Interactive effects of copper oxide nanoparticles and light to green alga Chlamydomonas reinhardtii. Aquat. Toxicol. 170, 120-128. https://doi.org/10.1016/j.aquatox.2015.11.018 (2016).

71. Baker, N. R. Chlorophyll fluorescence: A probe of photosynthesis in vivo. Annu. Rev. Plant Biol. 59, 89-113. https://doi.org/10.1146/ annurev.arplant.59.032607.092759 (2008).

72. Xia, J. \& Wishart, D. S. Web-based inference of biological patterns, functions and pathways from metabolomic data using MetaboAnalyst. Nat. Protoc. 6, 743. https://doi.org/10.1038/nprot.2011.319 (2011).

73. Jung, Y. et al. Characterization of dandelion species using $1 \mathrm{H}$ NMR- and GC-MS-based metabolite profiling. Analyst 136, $4222-$ 4231. https://doi.org/10.1039/c1an15403f (2011).

\section{Acknowledgements}

V.I.S. acknowledge the financial support of the Swiss National Science Foundation (Grant number IZSEZ0_180186). AK acknowledges the support of U.S. National Science Foundation (Grant NSF 1901515). Any opinions, findings, and conclusions or recommendations expressed in this material are those of the author(s) and do not necessarily reflect the views of the funding agencies.

\section{Author contributions}

V.I.S., W.L., SM. and A.K. conceived and designed the study. W.L. performed the AgNPs characterization, bioassays for physiological response assessment, exposure assays for metabolomics, analyzed the physiological response data and provided interpretations. W.L. performed the LC-MS measurements and data processing, S.M. prepared the samples for metabolomics and overviewed the LC-MS measurements. V.I.S. performed analysis and interpretation of metabolomics results, wrote the manuscript, overviewed the overall study. A.K. took part in the data interpretation, manuscript writing, overviewed the overall study. All the authors critically commented and revised the manuscript. All the authors have approved the paper submission.

\section{Competing interests}

The authors declare no competing interests.

\section{Additional information}

Supplementary information is available for this paper at https://doi.org/10.1038/s41598-020-77521-0.

Correspondence and requests for materials should be addressed to V.I.S.

Reprints and permissions information is available at www.nature.com/reprints. 
Publisher's note Springer Nature remains neutral with regard to jurisdictional claims in published maps and institutional affiliations.

(c) (1) Open Access This article is licensed under a Creative Commons Attribution 4.0 International License, which permits use, sharing, adaptation, distribution and reproduction in any medium or format, as long as you give appropriate credit to the original author(s) and the source, provide a link to the Creative Commons licence, and indicate if changes were made. The images or other third party material in this article are included in the article's Creative Commons licence, unless indicated otherwise in a credit line to the material. If material is not included in the article's Creative Commons licence and your intended use is not permitted by statutory regulation or exceeds the permitted use, you will need to obtain permission directly from the copyright holder. To view a copy of this licence, visit http://creativecommons.org/licenses/by/4.0/.

(c) The Author(s) 2020 